\title{
Problemas conceptuales, relaciones de trabajo y derechos laborales de los trabajadores informales
}

\author{
ENRIQUE DE LA GARZA TOLEDO
}

> Profesor-investigador del Postgrado en Estudio Laborales de la UAMI, Premio Nacional de Economía, Premio Nacional de Investigación Laboral, Premio Nacional de Ciencias y Arte; Postdoctorado en las Universidades de Warwick, Inglaterra y Berkeley, California. egt570@gmail.com

\section{Universidad de Valparaíso}

Facultad de Arquitectura

Márgenes

Espacio Arte Sociedad

Problemas conceptuales, relaciones de trabajo y derechos laborales de los trabajadores informales

Diciembre 2012, Vol. 9, N 11

pp. 162-168

ISSNN 0718-4034

Recepción: Agosto 2012

Aceptación: Diciembre 2012

\section{RESUMEN}

En este artículo se discute la necesidad de un concepto ampliado de trabajo, primero que no se reduzca al trabajo asalariado, segundo que tampoco se remita exclusivamente al trabajo que genera bienes o servicios para la venta; se relaciona lo anterior con la necesidad de un concepto de relación laboral que rebase a la obrero patronal, a través del concepto de relación social de producción, de esta manera es posible incluir en dicha relación a los trabajadores por su cuenta a consumidores y agentes no laborales en el espacio urbano. Finalmente, se cuestiona que el derecho laboral deba excluir a los no asalariados y a los de zonas oscuras de asalaramiento, por el contrario se propone recuperar experiencias de cambios legislativos de otros países que los incluyen dentro del derecho laboral, a través de conceptos como derechos mixtos, cuasipatrón.

PALABRAS CLAVE

Informalidad, servicio, derechos laborales

\section{Conceptual problems, working relationships and labor rights ABSTRACT}

This article discusses the need of a broader concept of work. First, one that is not reduced only to paid work. Second, a concept that does not refers only to the kind of work which produces goods or services. A new concept of social relation of production that goes beyond the employer-employee relationship is needed. Under this new conception of work, it is possible to incorporate independent workers, consumers and non-working agents in the urban space. Finally, the fact that labor rights fails to recognize unpaid workers, and those working under the shady areas of wage earning is questioned. Considering foreign experiences in which legal changes have been made to include them within labor rights is proposed. This has been made under concepts such as mixed rights, cuasipatrón.

KEY WORDS

Informality, services, labor rights

Des problèmes conceptuels, des relations de travail et des droits du travail des travailleurs informels

RÉSUMÉ

Cet article met en évidence la nécessité d'établir un concept plus vaste du travail, en première lieu que celui-ci ne se réduit uniquement au travail salarié, ni non plus au travail qui produit des biens ou services destiné a la vente; cela dû au besoin d'un concept de lien de travail qui dépasserait celui de ouvrierpatronat, grâce au concept de relation social de production, de cette manière il est possible d'inclure dans cette relation les travailleurs individuels aux consommateurs et agents non travailleurs sur l'espace urbain. Finalement, nous nous interrogeons le fait d'exclure les non salarié des droits des travailleurs et à ceux qui appartiennent au zones d'ombres des salariées, au contraire nous proposons récupérer des expériences de changement législatif d'autres pays qui les incluent dans le droit de travail, à travers le concept de droits mixte, quasi patronat.

MOTS CLÉS

Informalité, services, droit de travaille 
El concepto de informalidad ha sido definido de muchas formas (Tokman, 1987), hay quien afirma que hay 64 maneras distintas de definirlo, aunque pensamos que estas definiciones podrían agruparse en tres, con sus respectivas variantes (Tokman, 2006). Aunque, habría que aclarar que el problema de fondo de la definición de un concepto no son los indicadores apropiados para medirlo sino en primer lugar a qué problema responde la definición y, segundo, el contenido teórico del mismo, que implica también la relación con otros conceptos. Por lo tanto un camino equivocado para iniciar la discusión de la pertinencia o impertinencia del concepto de informalidad es empezar por una definición operacional, este es también un problema importante pero derivado hasta cierto punto del primero, sobre todo cuando las definiciones operacionales se ajusten al universo de variables disponibles a partir de encuestas representativas o censos previamente recabados por institutos como el INEGI. De esta manera el pragmatismo puede predominar en cuanto a hacer una definición solamente en términos de los datos disponibles, cuando se podría primero definir teóricamente el concepto y luego preguntarse si la estadística disponible satisface nuestra definición, bajo el principio de que nuevas variables podrían eventualmente ser incluidas en las encuestas. Asimismo, quienes definen los conceptos son los especialistas orientados por ciertos marcos teóricos, metodológicos e interesados en la solución de determinados problemas. Es decir, los conceptos no se autodefinen sino somos nosotros los que los definimos y en ciertas condiciones logramos que nuestras comunidades e instituciones los acepten.

Bajo esta consideración no haremos una Historia detallada de los cambios que ha tenido el concepto de informalidad, puesto que otros ya lo han hecho con propiedad (Rendón, 1991), sino que trataremos de partir de que la intencionalidad y contenido de la informalidad, más allá de los indicadores propuestos, pudieran agruparse en tres grandes tipos:

1. El original del grupo de la OIT en Kenya que a su interior también reconoció varias definiciones, sea como unidades productivas que no contratan mano de obra, no maximizan ganancias, ponen en juego escaso capital y tecnología, con procesos productivos de limitada división del trabajo y sin límites precios entre producción y reproducción en el ámbito de la familia o bien como sector productivo que genera bienes o servicios en pequeña escala, con procesos intensivos en mano de obra, calificada fuera del sistema educativo formal, que participa en mercados de trabajo no regulados, que produce con escasos recursos de propiedad familiar. Entrar al detalle tendría que suponer dilucidar primero por qué fue definido de esta forma y no como falta de protecciones laborales simplemente, por ejemplo, y cuál concepto convendría actualmente (Cortés, 2000). En cuanto al problema detrás del concepto inicial de informalidad hay que situarlo en la coyuntura de la Historia de las Teorías y la económica-política concreta de los años setenta. En cuanto a lo primero, lo que estaba detrás era la idea de lograr el Desarrollo de segmentos tradicionales hacia los modernos, y por lo que respecta a lo segundo, se partía de la constatación de la permanencia de estas unidades productivas en forma abundante en el mundo subdesarrollado a pesar de la extensión de las relaciones mercantiles. Es decir, el problema en el fondo era cómo salir del subdesarrollo, de tal forma que la unidad de análisis tendría que ser la unidad o establecimiento productivo que conformaba un sector con ciertas características. Estas características estaban centradas en la forma de producción, medios de producción, mano de obra, relación laboral, su relación con los mercados, es decir, la intencionalidad y el contenido se acerca mucho a lo que actualmente llamaríamos Modelo Productivo. No significa que fuera un enlistado exhaustivo de indicadores al que sólo faltara un nombre actualizado, frente al abandono de los conceptos de tradicional y moderno, sino que podría asimilarse al mismo. El concepto de Modelo de Producción data de los años noventa y no fue construido para dar cuenta de la informalidad sino de las diversas formas de producción en el marco de la reestructuración productiva arrancada desde los ochenta (De la Garza, 2007). Entre sus componentes cabe destacar el nivel de la tecnología utilizada, la forma de organización del trabajo, las relaciones de trabajo, el perfil de la mano de obra, las relaciones con el medio inmediato de la empresa tales como encadenamientos hacia delante y hacia atrás. La ventaja que tiene este concepto con respecto de tradicional o modernos es que es más analítico y lo que se llamaba moderno podría alcanzarse por diversas vías, de tal forma que el camino del desarrollo no sería necesariamente el de la industrialización como antes se pensaba (Gortz, 1991). En esta primera acepción el punto central no está puesto en la no regulación sino en las condiciones precarias de producción que se traducen en bajas productividades, calidades, falta de justo a tiempo, además de las laborales, que obstaculizan, a pesar de hallazgos posteriores que encontraron articulaciones tradicional-moderno, un desarrollo más sostenido y equilibrado (Sen, 2000).

2. El otro gran conjunto en la definición de informalidad es el que pone el acento en las relaciones laborales sin protecciones, este concepto se acerca a los actuales de no estructurado (INEGI, 2004), atípico (De Grip, 1997), desprotegido (Sennet, 2006), excluido (Pérez Sainz, 2003), vulnerable (Pizarro, 2001), inseguro (Castel, 2004), no decente (Reglias, 2003), no estándar (Marshall, 1987) en sus correlaciones con la precariedad (García, 2006). No entraremos a las distinciones finas entre estos conceptos, tampoco estamos afirmando que precario es su sinónimo, en este caso se trata de una relación estadística positiva entre los dos conceptos. Un grupo de definiciones como la mencionada es pertinente en cuanto a la existencia de un sector importante de trabajadores no protegidos, sea porque se violan las normas laborales o bien porque se encuentran en zonas obscuras de definición de sus relaciones de trabajo (De la Garza, 2005). Es el caso que abordaremos con mayor profundidad de los trabajadores por cuenta propia que a primera vista aparecen situados en relaciones no laborales al no contar con un patrón en el sentido tradicional, de tal forma que la demanda de cumplimiento de mínimos en condiciones de trabajo o seguridad social no serían pertinentes (De Buen, 1993).

3. Lo informal como unidad productiva para autoemplearse y generar ingreso reproductivamente, sin separación clara entre el funcionamiento y finanzas del hogar y del trabajo, que lleva a la definición operacional actual de informales como no registrados, sin usar una contabilidad racional que separe producción de reproducción (Portes, 1995). La primera parte de la definición pareciera apuntar hacia la número uno, pero la parte productiva es simplificada al extremo, de tal forma que no permite - tal vez porque no interesa- profundizar en estos modelos de producción, sino poner el acento en su no regulación como unidad más que en sus consecuencias laborales y en particular, al utilizar como indicador privilegiado la existencia de una contabilidad separada entre producción y reproducción pareciera dirigirse hacia el problema de la evasión fiscal y la falta de registro como unidad productiva, como problemas centrales.

Actualmente la oІт ha llegado a una definición dual, por un lado informalidad de la unidad productiva y por el otro lado informalidad de la 
ocupación, de tal forma que estas no coinciden y, en particular, los trabajadores de las unidades productivas informales son menores que los que trabajan en condiciones laborales informales.

Es decir, las tres definiciones son pertinentes, dependiendo del problema eje que se trate de resolver: el desarrollo sostenido, el trabajo decente o la evasión fiscal y el no registro. En otras palabras, la definición más adecuada pasa por definir mejor el problema que se trata de ayudar a resolver con las mediciones de informalidad. Los marcos teóricos también influyen, si en los setenta era legítimo el concepto de desarrollo, suponía las políticas públicas para impulsarlo, especialmente de políticas económicas e industriales, si estas concepciones cambiaron y se dejó al mercado el problema de crecer y de mejorar las condiciones de vida, era lógico que el concepto cambiara (Boltansky y Capello, 2002). Pero como el mercado resultó en nuestros países insuficiente para desarrollar y mejorar los niveles de vida y hay una permanencia de este tipo de actividades, quedaba el paliativo de dirigirlo hacia la violación de normas laborales y de extender la protección laboral y social a sectores no claramente asalariados. Pero si preocupaba el sector informal como competencia desleal del formal, cadena que iba del no registro al no pago de impuestos ni de gastos indirectos de mano de obra, luego el énfasis no era propiamente su erradicación en cuanto a modelo productivo sino en cuanto a su no regulación. Tampoco hay que pensar que las políticas públicas son siempre tan homogéneas teóricamente, las fallas del mercado y externalidades han llevado a reconocer cierto papel estatal en la promoción de los micro establecimientos, aunque se trata en general de paliativos por, frente a visiones más fundamentalistas que dejarían la suerte de estas a la acción depuradora del mercado (Bouffartigue, 1997).

\section{PROBLEMAS PREVIOS A LA DEFINICIÓN DEL CONCEPTO DE INFORMALIDAD DEL TRABAJO}

El concepto clásico de Trabajo debe ser reconsiderado, sin lo cual podría ser superficial llegar a un concepto diferente de trabajo informal. Este concepto clásico de Trabajo se refiere a este como mercancía (la fuerza de trabajo como capacidad para producir o circular mercancías) o bien al trabajo autónomo que genera bienes o servicios para el mercado. El primero es propiamente el trabajo asalariado que proporciona Trabajo a cambio de un salario a un patrón. Ambos implican una demanda, sea de fuerza de trabajo o bien del producto del trabajo independiente. Por lo tanto, en esta definición clásica si no hay mercado no hay Trabajo y no hay ocupación. Esta definición, como todas, no es algo natural, sino obedece a cierta intencionalidad y concepciones teóricas y visiones sobre la marcha de la sociedad. Da cuenta de Trabajos que contribuyen al valor agregado y, por lo tanto, tienen consecuencias en el PIB visto como riqueza social, tiene como visión una sociedad del mercado o regida por el mercado en la que cada quien recibiría según su contribución a la riqueza. Pero esta definición deja de fuera dos circunstancias, la primera es histórica, antes del capitalismo o de sociedades con mercados desarrollados, la riqueza principal la generaban esclavos y luego siervos. Tanto unos como otros no estaban sujetos a un mercado de Trabajo, el esclavo no vendía su fuerza de trabajo sino era vendido en su integridad como si fuera un animal, es decir era un medio de producción y no una fuerza de trabajo. No había propiamente un mercado de trabajo, ni lo producido era siempre vendido en un mercado sino dedicado a la subsistencia. Otro tanto sucedía con el siervo de los señores feudales en la edad media, se nacía siervo por coerción extraeconómica, se trabajaba las tierras del señor en parte para aquello y en parte para subsistir. Tampoco había un mercado de trabajo y el producto del mismo se dedicaba a la subsistencia. Es decir, habría que hacer muchos malabarismos conceptuales para incluirlos dentro del trabajo clásico capitalista que es el que actualmente predomina y, en esta medida, la realidad a la que alude el concepto clásico de Trabajo ha cambiado históricamente y seguramente nadie negaría que los trabajos del esclavo y del siervo deberían de ser considerados como trabajo, así como el que se desarrollaba en las comunidades primitivas para la subsistencia sin casi intercambios mercantiles.

El otro tipo de Trabajo no incluido en el concepto clásico es el que genera bienes y servicios pero no para el mercado, es el actual de autoconsumo, el doméstico no asalariado, el comunitario, etc. El lenguaje común también los designa como trabajo, aunque no se les incluya en las cuentas nacionales. Aunque hay razón en el lenguaje común cuando el concepto de Trabajo se amplía de uno que tiene que ver con el mercado a otro que lo incluye pero a la vez lo rebasa, ya que en su definición básica está el generar valores de uso que satisfacen necesidades de los hombres estén dirigidos al intercambio o no.

Aunque la definición clásica de Trabajo vinculado al mercado incluye al independiente que básicamente no contrata trabajadores asalariados y es realizado por el propietario con miembros de su familia cuando el producto se dirige a la venta, no hay duda que el concepto clásico privilegia sobre todo al trabajo asalariado, tal vez bajo la concepción dualista de que finalmente seríamos una sociedad de dos clases sociales, por la superioridad de la producción capitalista sobre la pequeña producción para el mercado. En esta medida, el concepto de Trabajo detrás del derecho laboral clásico es el asalariado. Esta reducción de la relación social de producción al concepto de relación laboral, como relación capital-trabajo, tiene razones históricas importantes. Con el desarrollo de la producción mercantil aparece propiamente la fábrica que no solo produce para el mercado y, en esta medida se diferencia del taller artesanal también por la introducción del maquinismo, resultado inicial de la revolución industrial, que implica mayor concentración de obreros en un espacio definido y la conformación del concepto de jornada laboral. Esta nueva clase trabajadora a través de sus luchas, organizaciones, partidos finalmente logró su reconocimiento como clase diferenciada y las normas de cómo trabajar, concepción que está detrás del derecho laboral que en forma desarrollada corresponde apenas al siglo XX. Derechos, deberes en torno a la relación laboral entre el capital y el trabajo, reconocimiento de esta situación social primero por el Estado y luego por los empresarios, llevaron a los conceptos de regulación, de derechos mínimos individuales y colectivos, de contratación colectiva, asociación, huelga y de seguridad social. Es decir, fueron las presiones de los trabajadores asalariados, sobre todo los organizados fabrilmente, las que lograron la conformación de los sistemas formalizados de derechos y obligaciones, así como las formas para dirimir las disputas, mediados por los Estados. Este ha sido el cuerpo clásico del derecho laboral y de la seguridad social como resultado histórico que pone el énfasis en el trabajo como trabajo asalariado.

Pero un concepto más básico de relación social de producción, anterior al de relación laboral, puede ser muy útil para la extensión del concepto a trabajadores no asalariados e incluso a trabajos que no se dirigen al mercado. El concepto básico de relación social de producción considera una definición muy amplia y relativamente abstracta primero de Trabajo: transformación de un objeto de trabajo utilizando medios de producción y fuerza de trabajo para generar un 
producto útil para satisfacer necesidades humanas. En el proceso de producción los hombres que participan se ponen en relación con medios de producción y objetos de trabajo pero también con otros hombres, estas relaciones sociales entre hombres en el trabajo serían las relaciones sociales de producción. Una forma particular de dichas relaciones serían de dependencia con un patrón pero en otras pueden ser entre miembros de la familia, entre un pequeño propietario que a la vez trabaja en forma directa con algunos asalariados y como veremos, en muchos servicios que no se pueden proporcionar sin la presencia del consumidor, directamente con este en el proceso de trabajo -el servicio de salud o de transporte personal no puede operar sin estar presente el consumidor al mismo tiempo que se genera dicho servicio (Camaño, 2005).

Una relación social en el trabajo entre quienes intervienen en el mismo, sean productores directos o no, implica interacción entre dos o más actores, interacciones que conllevan significados, posibilidad de acuerdos o desacuerdos, cooperación o conflicto. Es decir, toda producción tiene un aspecto simbólico desde el momento en que intervienen hombres. En unos casos la capacidad de simbolización y por tanto de dar significado al trabajo, a la relación de trabajo, al producto, quedará muy subordinada a las reglas de cómo trabajar -taylorismo-; en otras la capacidad de decisión del trabajador será mayor, pero en todas las formas de trabajo interviene la capacidad humana de construir significados. Por otro lado, las concepciones clásicas del trabajo han estado muy centradas en el trabajo material y específicamente con producto físicamente objetivable, separable del productor, en procesos productivos fabriles -con segmentación clara en tiempo y espacio de producción de los que no lo son-, maquinizados, con concentraciones apreciables de número de trabajadores, con una estricta división del trabajo, con administración científica, contable y financiera, con sindicato, contrato colectivo.

La producción material con producto físicamente separado de los productores, que puede ser almacenado y revendido fue hasta los años sesenta el eje de la producción moderna, capitalista, pero la situación empezó a cambiar desde esos años a favor de los servicios. Para estos tradicionalmente se ha utilizado el concepto de producto intangible, pero este resulta muy poco analítico y poco preciso ante la irrupción masiva de lo que no es industrial. Hay servicios en donde una parte del producto es perfectamente tangible como el platillo en un restaurante, en cambio hay intangibles que se parecen más a la producción material porque el producto existe al final independiente de su creador como el programa de software. De tal forma que de la clasificación entre productos tangibles y no tangibles tal vez hubiera que pasar al de objetivados y no objetivados (subjetivados). La objetivación es un concepto fiIosófico que puede venir al caso, es decir, productos del trabajo que adquieren una existencia separada de sus creadores. Estos productos pueden estar físicamente objetivados como un automóvil pero pueden ser meramente simbólicos, como el programa de software. Un programa de software no es sino un algoritmo -combinación de símbolos-que sirven para resolver un problema, pero, a diferencia de la simbolización que todos hacemos en nuestros mundos internos para relacionarnos, estos símbolos están objetivados. Es diferente de los servicios que necesitan forzosamente del cliente-consumidor en el momento de la producción del servicio -salud, educación convencional, transporte de personas, restaurante convencional, espectáculo en vivo, etc. En estos servicios, se opera una suerte de producción inmaterial, en cuanto a que el producto no existe objetivado sino que se incorpora directamente durante su producción a la subjetividad o al cuerpo del cliente, no se puede revender ni almacenar. La presencia del cliente durante la producción introduce la complicación de un tercer actor, cuando hay una relación laboral clásica, que no es patrón ni obrero pero que es indispensable para que se produzca. La complicación de un tercero en el proceso de trabajo puede extenderse a los trabajos que se realizan en espacios abiertos a la ciudadanía, como la venta callejera, el servicio de taxi o micro que ponen en relación durante la jornada de trabajo no sólo al trabajador con el cliente, sino con actores no laborales (automovilistas, agentes de tránsito, inspectores, policías, transeúntes, etc.) que sin tener un objetivo laboral o de compra y venta intervienen o interfieren, ayudan u obstaculizan en la actividad laboral in situ ${ }^{1}$.

Una complicación semejante se genera cuando producción y reproducción se traslapan en tiempo y espacio, como en el trabajo a domicilio, porque el traslape es también de relaciones entre actores que trabajan y otros que reproducen su vida desde el momento en que comparten espacio y tiempo.

Asimismo, lo intangible queda corto frente a la carga del contenido simbólico en el propio producto, desde aquellos productos reducidos a lo simbólico, objetivados (programa de software) o subjetivados (solo existen en el momento de su producción frente a los consumidores como el espectáculo en vivo), que pueden tener énfasis diversos en lo cognitivo (software), emotivo (cuidado de ancianos), estético (moda) o moral y que suponen calificaciones a veces muy diferenciadas de quienes realizan estos tipos de trabajo vinculadas con capacidad de interacción con otros, en especial con los clientes ${ }^{2}$. Otra complicación adicional sería la diferencia entre quien trabaja cara a cara, sea en forma clásica frente a clientes y otros actores, del trabajo con relación virtual en call centers, teletrabajo, etc.

Todas estas consideraciones deberían de llevar hacia un concepto ampliado de trabajo, no reducido al asalariado fabril con relaciones formales. Sin ir tan lejos como considerar trabajo al que no tiene vínculo con el mercado, ya resulta de suficiente interés el trabajo independiente para el mercado, pero también la profundización del carácter de las relaciones de trabajo en los servicios mercantiles que pueden implicar producción inmaterial (obra de teatro), participación de agentes no clásicamente laborales en el proceso de producción (taxis, micros, venta ambulante), la desterritorialización del espacio de trabajo (venta de productos a domicilio), los traslapes entre producción y reproducción (trabajo en casa), la relación puramente virtual de trabajo con los clientes y, a veces, con la jerarquía de la empresa (teletrabajo, call center) (Holdman, Batt y Hotkgrove, 2007). En términos de regulaciones esta profundización puede transformar conceptos básicos como el de cuándo hay una relación laboral, entre quiénes se establece, qué es jornada de trabajo y lugar de trabajo, quiénes son sujetos de derecho laboral.

Un concepto especialmente importante cuando queremos tratar el de derechos laborales es el de relación laboral, que como mencionamos quedó históricamente reducido a los vinculados entre el capital y el trabajo, dejando fuera todo tipo diferente de trabajo, pero más aún, muy centrado en la relación laboral clásica de fábrica - espacio cerrado y jornada laboral precisa- que permite delimitar claramente el tiempo y el espacio en que se realiza la relación laboral, sin intromisión de clientes ni otro tipo de actores en dicha relación laboral, ni mucho menos el traslape con la familia y en la que el problema del control sobre el espacio de 
trabajo no es particularmente importante porque queda definido por la subordinación (Castells, 1999). En cambio, cuando se pasa, como proceso de abstracción, del concepto de relación laboral, al de relación social de producción resulta que los otros trabajos no clásicos también implican relaciones sociales durante el proceso de producción, no sólo entre patrones y asalariados, sino con otros agentes, incluyendo a los clientes, de tal forma que aparece el problema también de las interfases o zonas oscuras entre aspectos implicados en las relaciones laborales clásicas con otras de carácter urbana, sanitarias, de tránsito, etc. (Yepes del Castillo, 1994). Que puede llevar a tres concepciones sobre el derecho, primero, la más tradicional que es conservar la esfera de cada derecho por separado, laboral, comercial, civil, etc. La segunda es la idea de lo mixto, reconocer que puede incumbir a varias ramas del derecho un acto laboral, aunque no queda claro en la actual distribución por códigos y tribunales como se combinarían sin caer en la primera posición, y la tercera, la más innovadora, que implica considerar dentro de un derecho aspectos que aparecían como de otra esfera de las regulaciones (Castillo y Orssati, 2005). Este último planteamiento coincidiría con un concepto ampliado de relación laboral que puede trascender la bilateral y convertirse en multilateral. Por lo pronto una primera ampliación del concepto de trabajo y de relación social de producción podría ser hacia el trabajo por su cuenta, que coincide con la definición de la oir de trabajo decente, que no es solo para el trabajo asalariado (Barreto, 1999).

\section{SEGUNDA VUELTA A LA INFORMALIDAD}

Sí el interés fuera la relación entre informalidad y derechos laborales se podría abordar de la siguiente manera. Primero, analizando los tipos de trabajos que actualmente se incluyen en el concepto de informalidad y dependiendo de sus características en qué aspectos podría estar sujeto a derechos y obligaciones. El sector informal, en cualquiera de sus definiciones implica relaciones sociales de producción en el sentido ampliado que hemos manejado. En esta población trabajadora informal habría que distinguir entre:

1. Los estrictamente asalariados, los trabajadores no remunerados, comúnmente parte de la familia y los de percepciones no salariales. Estos pueden ser de planta, por tiempo y obra determinados, discontinuos, ocasionales, subcontratados, por comisión. Es decir, un primer reto es reformar la legislación para que las zonas oscuras se aclaren, por ejemplo que los trabajadores por comisión sean considerados como asalariados y hacer cumplir las normas laborales. Lo anterior estaría en contra de tener dos niveles de derechos, unos para empresas medianas y grandes y otros para pequeñas y micro. Es cierto que las capacidades financieras y para cumplir obligaciones laborales entre estas son diferentes, sin embargo, los derechos laborales mínimos que garantiza la Ley Federal del Trabajo son suficientemente bajos como para pensar que podrían disminuirse más y conformar por Ley dos tipos de pisos laborales. La exigencia de eficiencia en las empresas para permanecer en el mercado debería de extenderse a otra de poder cumplir con normas laborales so pena de desaparecer. Es cierto que en este tema hay dos posiciones y algunas legislaciones consideran obligaciones menores de tipo laborales para las pequeñas empresas. Se trata de un debate que hay que continuar.

2. Otra situación se presenta con los autoempleados y pequeños propietarios que trabajan (el $80 \%$ de los taxistas en el D.F. o la mayoría de los vendedores ambulantes lo son). En estos casos la ley laboral tendría que modificarse para incluirlos, en parte recono- ciendo como formas laborales de subordinación las que aparecen como mercantiles entre empresas grandes y pequeñas cuando hay subcontratación de los servicios de sus trabajadores. Contratos mercantiles que a veces deberían de ser considerados en su aspecto laboral en tareas de maquila en donde el concepto clave puede ser el de quien tiene el control de las materias primas, de la maquinaria, de la tecnología, de la organización, de las características de la mano de obra y, por supuesto, del producto, para replantear con respecto a quien un trabajador es subordinado.

Lo anterior requiere de la ampliación del concepto de trabajo subordinado que puede ser entre empresas mercantiles, pero también incluir la situación de las agencias de contratación de personal y las cooperativas de trabajo, formas que a veces se utilizan para eludir responsabilidades laborales (De la Garza, 2005). En otros casos, cabe discutir el nuevo concepto de cuasi-patrón, para referirse a las relaciones entre unidades económicas con el Estado, como serían los trabajadores ambulantes, taxistas, microbuseros, que implicaría la noción de derechos frente al Estado, incluyendo la seguridad social, con la ampliación del concepto de sindicato al de no asalariados.

3. Decíamos que una complicación en los casos anteriores se presenta cuando intervienen otros actores fuera de los tradicionales o solamente frente al pequeño propietario en el trabajo. Esta situación se puede dividir en dos, en espacios cerrados a la ciudadanía con excepción de los clientes que compran el servicio o el bien y, la segunda, cuando el trabajo se realiza en espacios abiertos a la ciudadanía. La diferencia es entre una relación en el trabajo que puede ser triádica (propietario-asalariado-cliente) o bien propietario-cliente, a otra con intervención de los explícitamente interesados en la producción o el producto asimilable a lo anterior, más la intervención circunstancial de la ciudadanía que se mueve en el territorio público y agentes que intervienen sin que tengan que ver legalmente con lo laboral. Se trataría de los traslapes entre normas por esferas del derecho, que sin embargo forman parte de la misma relación de producción y que no deberían de separarse. En esta medida las normas laborales para estos trabajos deberían de incluir, en primer término, los derechos y obligaciones del cliente e instancias de regulación y negociación mixtas. Pero, no podrían dejarse de lado los derechos y obligaciones de transeúntes, vecinos, salud pública, orden, etc.

Un tema especialmente importante a incluir en el derecho laboral de quienes trabajan en espacios públicos es el derecho y su limitación en el uso de dicho espacio puesto que este resulta un medio de producción fundamental en este tipo de trabajos.

4. Con respecto de los derechos colectivos, en el caso de asalariados y otros asimilables a estos del sector informal simplemente habría que vigilar su cumplimiento, flexibilizando los requisitos para pertenecer a cada tipo de sindicato que considera le Ley actual. Entre los trabajadores independientes se tendría que ampliar como dijimos el concepto de sindicato (sindicatos de trabajadores por su cuenta) y especificar las instancias de negociación de los mismos dependiendo de sus características - por ejemplo las mesas de negociación entre vendedores ambulantes y gobierno, así como la posibilidad de formación de federaciones y confederaciones con sindicatos clásicos ${ }^{3}$.

Habría que aclarar que en el plano internacional ya hay un buen camino recorrido en este proceso de ampliación del derecho laboral hacia los no asalariados y a las zonas obscuras de las relaciones laborales. En América Latina en Chile, Perú, Brasil, Panamá, Colom- 
bia, Venezuela, Ecuador y Uruguay se ha ampliado el concepto de sindicato hacia estos sectores, el concepto de cuasi-patrón está en la legislación paraguaya. Cambios en este sentido pueden encontrarse en Alemania, España y otros países europeos.

Una lista incompleta que proviene de estas experiencias en otros países en cuanto a derechos de los no asalariados y de sus asalariados cuando sea el caso en el sector informal sería:

\section{A la capacitación}

2. Reducción de costos de registro y simplificación de trámites para estas unidades

3. Reducción de impuestos

4. Simplificación en derechos de propiedad

5. Reducción en los costos de los contratos

6. Sindicalización de no asalariados y de trabajadores de zonas obscuras

7. Fomento de cooperativas

8. Extensión de la seguridad social en cuanto a seguro médico, por accidentes de trabajo, por desempleo, vivienda, maternidad 9. Créditos

10. Regulación de las empresas familiares en cuanto jornadas, días de descanso, trabajo nocturno, infantil.

En México un avance lo constituyó el Reglamento para el Trabajo no Asalariado del D.F. primero porque la problemática de estos trabajadores la remite a una instancia laboral y no de otra índole, la Dirección General de Trabajo y Previsión Social. Segundo, porque define al trabajador no asalariado como aquella persona física que presta a otra un servicio personal ocasionalmente. Aunque se queda corta la definición porque también se pueden producir bienes, además de servicios y no necesariamente puede ser ocasionalmente, debería de valer también para una relación más permanente y no toma en cuenta que en un 30\% los trabajadores en el sector pueden ser asalariados de los primeros. Además, el reglamento está demasiado centrado en la venta ambulante y servicios en espacios públicos, dejando fuera a los que se prestan en espacios exclusivos para el cliente. Aunque reconoce el derecho de asociación de estos trabajadotes en la forma de Uniones, las reglamenta al detalle siguiendo en su registro, estatutos, elecciones, asambleas a las reglas que establece la Ley Federal del Trabajo para los sindicatos. Sin embargo, se queda atrás al no incluir las críticas al corporativismo que se presenta con intensidad en estos sectores, al autoritarismo de los líderes y su falta de democracia, en particular se olvida de incluir el voto directo y secreto para elegir dirigente y la transparencia en negociaciones y finanzas. Finalmente contempla proporcionar servicio médico a los trabajadores registrados en una clínica dependiente del gobierno del D.F.

Sin duda que se trata de un avance que debería de contemplar a los que no trabajan en espacios públicos, aunque no diferencia entre trabajo familiar de los que trabajan como asalariados en forma continua, y no incluye los derechos del cliente, favorece implícitamente al corporativismo en las organizaciones de estos trabajadores.

\section{CONCLUSIONES}

1. La persistencia del concepto de informalidad no debería de sesgarse hacia aspectos recaudatorios sino incluir información de la informalidad como modelo de producción y también como relación laboral que se aparta del trabajo decente, encaminada esta última hacia reformas laborales que consideren derechos de los informales en sus diversas modalidades (Portes, 1995)
2. Hay que reconocer en cuanto a derechos la heterogeneidad entre los que trabajan en el sector, primero a través de un concepto ampliado de trabajo y en especial de relación social de producción, que incorpore al cliente cuando sea pertinente y a otros actores no laborales: trabajadores asalariados/autónomos; en espacio cerrado a los clientes/en espacio cerrado a la ciudadanía pero no a los clientes/en espacios abiertos a la ciudadanía/en el hogar; con punto fijo de trabajo/desterritorializado; material/inmaterial; reconocer los trabajos de zonas oscuras y analizar la conveniencia de salariarlos jurídicamente.

3. Reconocer las zonas de derechos cruzados, laborales y no laborales y sus intersecciones.

4. Que los trabajadores no asalariados pueden ser sujetos de derechos y obligaciones, no necesariamente iguales o en los mismos rubros que los asalariados, pero los puede haber individuales y colectivos y de seguridad social.

5. Incorporación del concepto de sindicatos de trabajadores no asalariados y flexibilizar los tipos tradicionales de sindicatos contemplados en la Ley para facilitar la incorporación de trabajadores de las zonas oscuras del asalaramiento.

6. Que el proceso de reconocimientos de derechos es también un proceso de formalización que empieza por el registro individual del trabajador informal ante las instancias pertinentes, de sus sindicatos con incentivos materiales, con capacidad gubernamental de negociación, definiendo legalmente las instancias encargadas de esto. Todo esto no para mantener la precariedad en la que se encuentran la mayoría de los informales sino para ayudarles a transitar hacia modelos de producción más productivos que implicarían políticas económicas más inclusivas de las unidades pequeñas y micro, en especial del sector informal.

7. En cuanto a indicadores necesarios para quienes podrían tomar decisiones en este sector (gobierno, Uniones de trabajadores, ciudadanía, partidos, empresarios, etc.) se podrían combinar los tres criterios mencionados en las definiciones del concepto: como modelo de producción, como relación laboral y su distancia con el trabajo decente y la recaudatoria, en particular:

7.1. Número de asalariados, familiares y con percepciones no salariales, a comisión y sus condiciones de trabajo.

7.2. Trabajadores propietarios y por su cuenta, diferenciados de gerentes o empresarios y sus condiciones laborales.

7.3. Los que trabajan en relación directa con el cliente o a través de medios electrónicos.

7.4. Los que trabajan en locales cerrados, o en abiertos solo a la clientela, y en espacios públicos, o bien en la habitación o sin territorio específico.

7.5. Los del trabajo material e inmaterial.

7.6 La pertenencia a organizaciones aunque no sean sindicatos.

7.7. Las formas de regulación del trabajo, aunque no hayan contratos colectivos ${ }^{4}$.

7.8. Capacitación.

7.9. Seguridad Social.

\section{NOTAS}

1 Hay reflexiones actuales sobre este tipo de trabajos, en Mac Donalds o en Wal Mart, que hablan de cómo la organización tradicional del trabajo por parte de las gerencias se extiende al propio cliente. Si el cliente no "trabaja" escogiendo los productos en el 
supermercado $u$ ordenando de acuerdo con un menú simplificado y preestablecido para no perder tiempo en el Mac Donalds, recibiendo el alimento y descargando los desperdicios en recipientes, el servicio no se genera cabalmente. Hay quien utiliza incluso el concepto de taylorización del cliente (De la Garza, 2010).

2 La sociología del trabajo ha adoptado legítimamente los conceptos de trabajo emocional que incluye como parte importante de lo que se vende el despertar emociones agradables en la clientela (cuidado de bebés por ejemplo), hay quien usa el concepto de fábrica de sonrisas y más recientemente el de trabajo estético para aquellas actividades no necesariamente artísticas en donde lo principal que se genera y se vende es belleza.

3 En México es frecuente que quienes trabajan en los espacios públicos formen organizaciones, cuando se formalizan toman sobre todo la modalidad de Asociaciones Civiles. Pero esta figura jurídica tiene muchas limitaciones y en la práctica funcionan como sindicatos de no asalariados que impactan a los asalariados de cada sector.

4 La definición llana del sector informal como no estructurado presenta problemas, primero porque una parte de estos trabajadores están registrados por instancias gubernamentales, así como sus asociaciones; segundo porque muchos cuentan con reglas de cómo trabajar provenientes de negociaciones ad hoc con autoridades de gobierno e incluso las Uniones de trabajadores que tienen reglas formalizadas; finalmente, porque la parte codificada se complementa con muchas reglas no escritas para el ingreso, funcionamiento y salida del Trabajo.

\section{BIBLIOGRAFÍA}

BARRETO, H. (1999) “Conceptos y dimensiones del Trabajo Decente”, Boletín CINTRAFOR, No 151. Lima: OIT.

BOLTANSKY, L. y E. CHAPELLO (2002) El Nuevo Espíritu del Capitalismo. Madrid: Akal.

BOUFFARTIGUE, P. (1997) “¿Fin del Trabajo o Crisis del Trabajo Asalariado?”, Sociología del Trabajo, No, 29. Madrid: Siglo XXI.

CAMAÑO, P. (2005) “Las Transformaciones del Trabajo, la crisis de la relación laboral normal y el desarrollo del trabajo atípico”, Revista de Derecho, julio, v. 18 , No 1.

CASTEL, R. (2004) La Inseguridad Social. B.A.: Manantial.

CASTELLS, M. (1999) La Era de la Información. México, D.F.: Siglo XXI.

CASTILLO, Gerardo y A. ORSSATI (2005) Estrategias de Sindicalización de "Otros" Trabajadores. Perú: OIT.

CORTÉS, F. (2000) "La Metamorfosis de los Marginales: la polémica sobre el sector informal” en Tratado Latinoamericano de Sociología del Trabajo. México, D.F.: FCE.

DE BUEN, Nestro (1993) Organización y Funcionamiento de los Sindicatos en Derecho Sindical. Madrid: Laia.

DE GRIP. J. HOEVENBERG y E. WILLEMS (1997) “Athypical Employment in the European Union” International Labor Review, V. 136, No 1, primavera.

DE LA GARZA, E. (2005) “Antiguas y Nuevas Formas de Subcontratación” en FESMEX ¿Relaciones Triangulares de Trabajo, fin de la estabilidad Laboral? México, D.F.: FES.
---. (2007) "Del Concepto Ampliado de Trabajo al de Sujeto Laboral Ampliado" en Teorías Sociales y Estudios del Trabajo. Barcelona; Anthropos.

-_-. (2010) Trabajo no Clásico, Organización y Acción Colectiva. México, D.F.: Plaza y Valdés-UAM (en prensa, una versión preeliminar puede consultarse en http://www.izt.aum.mx/alast)

GARCÍA, B. (2006) "La Situación Laboral Actual: marcos conceptuales y ejes analíticos pertinentes”, Trabajo, No 3, diciembre. México, D.F.: OIT-UAM.

GORTZ, A. (1991) Metamorfosis del Trabajo. Barcelona: Fontamara.

HOLDMAN, D., A. BATT y U. HOTKGROVE (2007) "The Global Call Center Report. Ithaca: Cornell University.

INEGI (2004) La Ocupación en el Sector no Estructurado en México. Aguascalientes: INEGI.

MARSHALL, A. (1987) "Non-standard Employment Practices in Latin America". Ginebra: OIT.

PÉREZ SAINZ, J.P. (2003) “Exclusión Laboral en América Latina”, Sociología del Trabajo, No 47.

PIZARRO, F. (2001) “La Vulnerabilidad Social y sus Desafíos”. Chile, CEPAL.

PORTES, A. (1995) En Torno de la Informalidad. México, D.F.: Miguel A. Porrúa.

REGLIAS, Ido (2003) “Lavoro Atipici, Nuovi, non Standard”, Sociología del Lavoro, No 97.

RENDÓN, T. (1991) El Sector Informal Urbano. México, D.F.: STyPS.

SEN, Amartha (2000) "Trabajos y Derechos", Revista Internacional del Trabajo, v. 119, No 2 .

SENNET, R. (2006) La Cultura del Nuevo Capitalismo. Barcelona: Anagrama.

TOKMAN, V. (1987) El Sector Informal Hoy. Chile: OIT.

---. (2006) Una Voz en el Camino. México, D.F.: FCE.

YEPES DEL CASTILLO, I. (1994) "Estudios Comparados de la Exclusión Social”, Revista Internacional del Trabajo, v. 113, № 5-6. 